\title{
Studying the candida resistance and sensitivity for some antifungals
}

\author{
Humam saadi Hussein Batol Imran Dheeb* Thekra Ahmed Hamada** \\ Biology Department / College of Science / University of Tikrit \\ *Biology Department /College of Education / University of Samaraa \\ **College of Medicine / University of Tikrit \\ E-mail:dr.humambio@gmail.com
}

\begin{abstract}
Back ground: The current study included 150 samples from patients with Candida from a hospital of Medical City / education labs - Baghdad, and ranged in age from $(10$ - 65)years for both sexes, all isolates taken from different sources (Lower respiratory tract, Urine, Skin, Vaginal and Oral thrux), where the number of males (55)as a percentage (37\%) while the number of females (95) as a percentage $(63 \%)$.

The objective :The objectives of this study are studying resistance and sensitivity of candida spp to some antifungals.

Material and methods: All the required material which used for the study, and the direct examination was conducted using potassium hydroxide KOH $10 \%$, As well as microscopic examination and laboratory transplantation for all samples, for the purpose of investigating the candidiasis. Also Vitek System examination was conducted on all positive samples for microscopy and laboratory culturing; and so to be diagnosed on the species level, and this study included using (3) anti-fungal equipped from Himedia (India), an (Fluconazole, Clotrimazole and Nystatin), anti-fungal used by standard disk Diffusion to know the resistance of Candida and its sensitivity toward used anti-fungal.

The results: The results of direct examination were vaginal swab (50), Sputum (28), Oral swab (34), Urine (21) and Skin swab (17).

Conclusion: Candidiasis was more common in female patients as well as from medical units, where the rate of infection in females more than in males, Candida species were identified by the manual and automated methods and we found that the automated method by using VITEK ${ }^{2}$ YST Card was the best for species identification.
\end{abstract}

Keywords: Antifungal, Candida Spp, Resistance, Sensitive, Fluconazole, Clotrimazole , Nystatin.

\section{Introduction}

Candidiasis is a fungal infection caused by yeasts that belong to the genus Candida. This infections may be primary or secondary fungal infection caused by Candida species, And generally all this infections may be acute, Sub acute, or chronic. There are about approximately 200 different species of Candida species but there are over twenty species of Candida yeasts that can cause infection in humans. It is worth mentioning fungal pathogen found as part of the normal microflora in the human digestive tract. [1,2]

The most common of which is Candida albicans and then followed by other species such as C.glabrata, C.krusei, C.norvegenesis, C.kefyr, C.parapsilosis, C. metapsilosis, C. orthopsilosis, C.famata, C.sphaerica, C.guilliermondii and others. [3, 4, 5].

All humans are colonized with Candida species, mostly Candida albicans yeasts which usually found and that naturally colonizes the in the intestinal or gastrointestinal tract and can be found on mucous membranes, skin and reproductive tract without causing infection; however, overgrowth of these organisms can cause symptoms to develop. Symptoms of candidiasis vary depending on the area of the body that is infected. Therefore we all carry this organism on our skin, in our mouth, in our gastrointestinal tract (gut), and, in the case of women, in the vagina. [1, 2, 5, 6, 7, 8].

Candidiasis may also infect the blood stream of human or internal organs such as the spleen or liver. In addition candidiasis is the most commonly identified Candida species in clinical contexts and is one of the leading causes of hospital-acquired infections. [6, 9, 10].

In recent years, the number of cases of fungal infections has increased. This is due to an increase in the number of diseases that weaken the immune system, and according to recent statistics fungi cause more than billion skin infections, One hundred [100] million mucosal infections, 10 million serious allergies and more than a million deaths each year. Global mortality owing to fungal infections is greater than for breast cancer, malaria, HIV and tuberculosis. [11, 12, 13].

During the last decades was noticed that the extravagant advances in modern therapeutic technologies has not only prolonged the life of critically ill patients but also has led to an enormous increase of fungal infections, This recent upsurge in fungal infections has resulted in high morbidity and mortality rate in the infected patients, and because of the various fungal infections and their spread, drugs and antifungal have 
been used to test infections. However, there has been a virulence factors in a used candida species resistance to antifungal which used and the failure of some antifungal to inhibition of growth in culture media.

Therefore, fungal infections that are resistant to treatment are a public health challenge. We all should have a role in preventing these infections and reducing antifungal resistance. [2, 14, 15, 16, 17].

The present study is directed to use of some antifungals fuconazole, nystatine and cotrimazole to determine the sensitivity of these species and resistance.

\section{Materials and methods \\ Samples collection:}

The swaps of vaginal and skin, sample of urine, sputum and lower respiratory were collected from 150 patients $(10-65)$ years old of both gender suspected have infection with Candidiasis, (as clinically identified by a physician), during the period of November/2017 to March/2018 from skin and respiratory diseases specialized center, Ministry of health, Baghdad Province. The samples were examined directly under the microscope using $10 \% \mathrm{KOH}$ and culturing on the sabouraud dextrose agar [18].

\section{Samples Culturing}

Samples were cultured on sabouraud dextrose agar (SDA) supplemented with $0.05 \mathrm{mg} / \mathrm{ml}$ chloramphenicol to inhibit the growth of bacteria, then incubated at $25^{\circ} \mathrm{C}$ and $37{ }^{\circ} \mathrm{C}$ for 10 days [19].

\section{Identification of Isolate Using direct microscopic examination [KOH]}

Microscopic examination was conducted using potassium hydroxide (KOH) $10 \%$, according to the method that mentioned [18].

\section{Development and diagnose isolates}

The isolates were cultured and diagnosis as stated in [20] for examination and diagnosis of the isolates.

Cultivation of samples on the media culture

Swabs were taken cultured on the sabouraud dextrose agar (SDA) in a glass dish, according to the method [18].

\section{Diagnosis of the genus Candida}

The special examination were Conducted for the diagnosis of Candida genus and their types and these examinations include the following:

- Phenotypic examination: phenotypic examination was conducted according to the method [21].

- Microscopic examination: microscopic examination was conducted according to what is stated in [19].

- Isolates development on the media of CHROMO agar: The isolates development on the media of CHROMO agar, according to [22].

- Surface growth formation examinations

This examination was conducted inoculating tubes containing liquid (SDA), as stated in [23].

- Germ tube formation of Candida albicans

Candida albicans formed germ tube after three hours of Incubation in human serum are shown in. Candida albicans showed a short tube like structure, with no constriction at the attachment point. This extension represents the beginning of true hyphae formation. No germ tube was observed in Candida species other than C. albicans.

\section{Diagnosis using biochemical test (Vitek apparatus)}

Diagnosed by genus and species using biochemical tests by Vitek System consists of Cassette and Reagent Cards that contained [64] pits every pit represents the substrate to conduct the test, and plastic pipes as well as Densi Chek device and the unity of the input and output of information [24].

\section{Results}

The results that showed in (Table 1) showed different of distributed more than one types of sample

Table (1): Distribution of Candida infections in affect clinical samples

\begin{tabular}{|l|c|c|}
\hline Sample & No. of isolates & Percentage\% \\
\hline Vaginal swab & 50 & 33 \\
\hline Sputum & 28 & 19 \\
\hline Oral swab & 34 & 23 \\
\hline Urine & 21 & 14 \\
\hline Skin swab & 17 & 11 \\
\hline
\end{tabular}




\section{Microscopic Exanimation}

The results of direct microscopy were shown in (Table 1) using $(10 \% \mathrm{KOH})$ and shown in the (Figure 1, 2, 3) [140] positive samples and (93\%) of the total number of samples of patients, while the number of negative samples by direct microscopy (10) samples and (7\%). And the number of male positive samples [48] isolation by (34\%) from total of positive isolations, while the results showed that the number of positive isolates for females (92) (66\%) from the positive samples total. In the negative cases, the results of the direct examination showed that the percentage of male negative samples (7) by [70\%] and female (3) by (30\%) from the negative samples total.

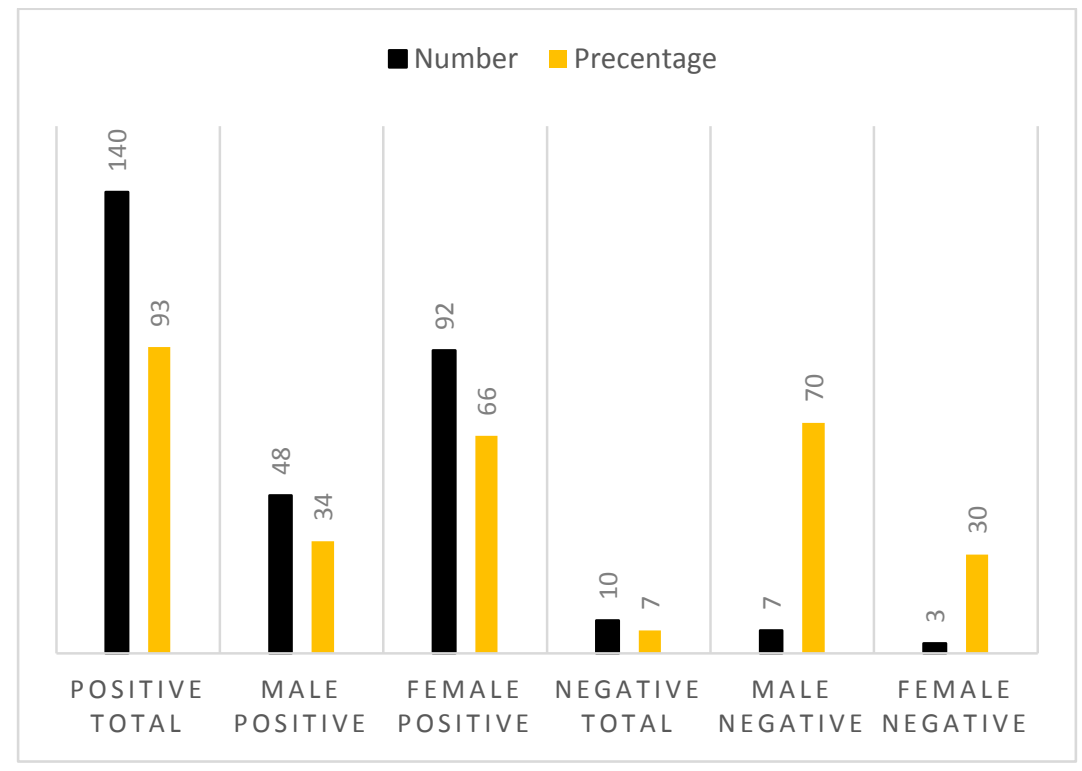

Figure (1): Results of direct microscopy using (10\% KOH). Surface growth formation examinations

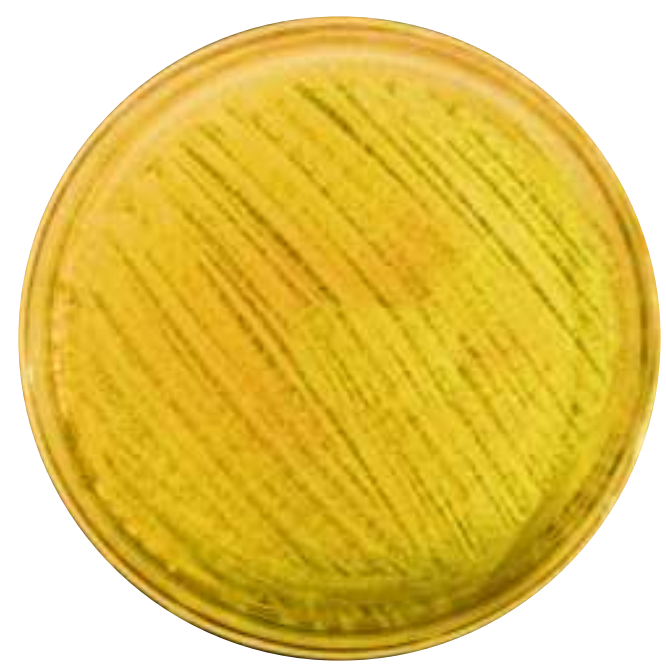

Figure (2): Growing Candida spp on Sabouraud dextrose agar after incubation for 24 hrs and at $35^{\circ} \mathrm{C}\left( \pm 2^{\circ} \mathrm{C}\right)$ 


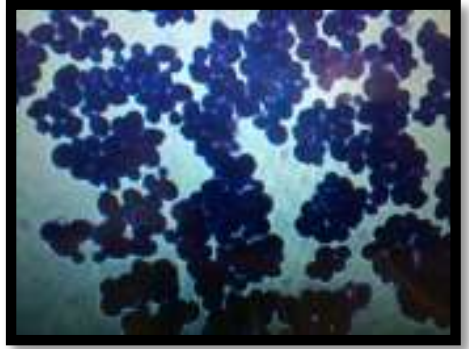

Microscopic picture of Candida albicans Using Gram stain

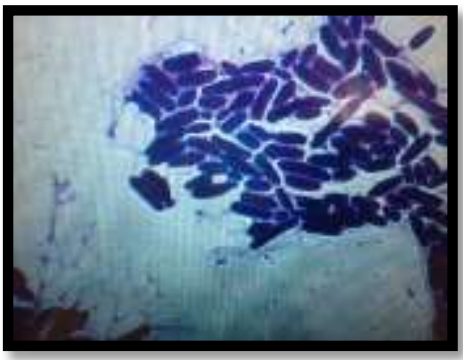

Microscopic picture of Candida glabrata Using Gram stain

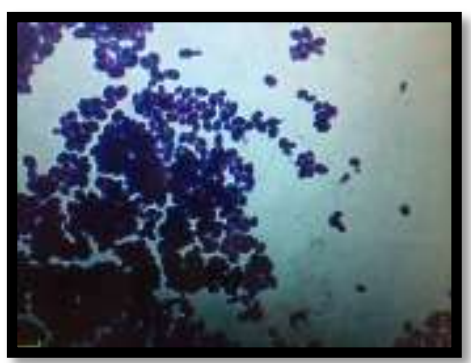

Microscopic picture of Candida kefyr Using Gram stain

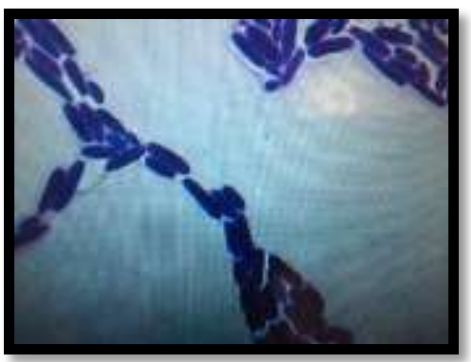

Microscopic picture of Candida norvegenesis Using Gram stain

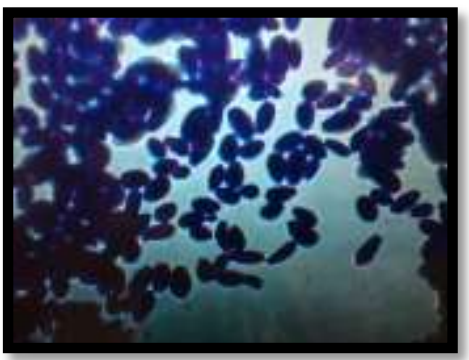

Microscopic picture of Candida sphaerica Using Gram stain

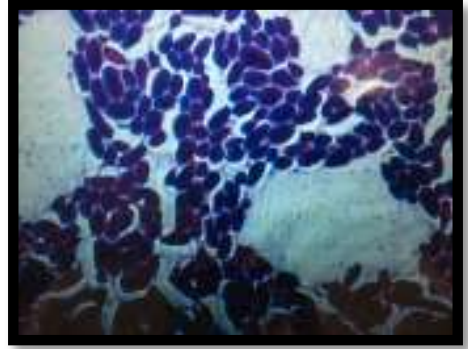

Microscopic picture of Candida famata Using Gram stain

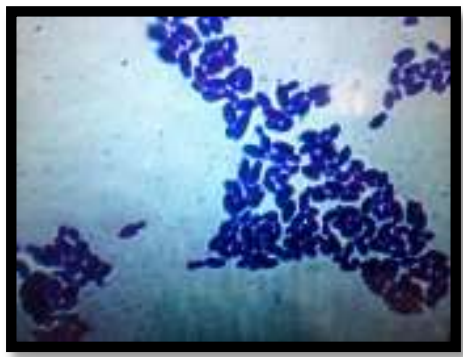

Microscopic picture of Candida guilliermondii Using Gram stain

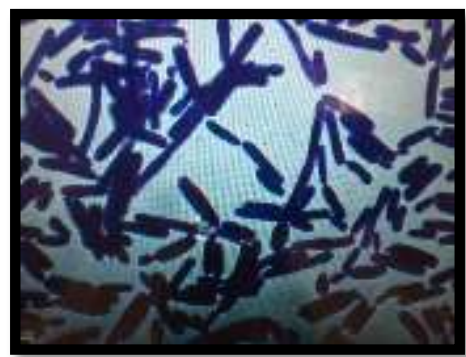

Microscopic picture of Candida krusei Using Gram stain

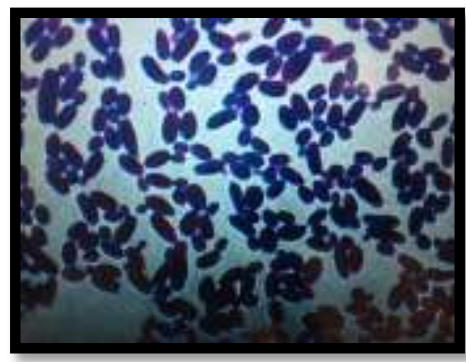

Microscopic picture of Candida parapsilosis Using Gram stain

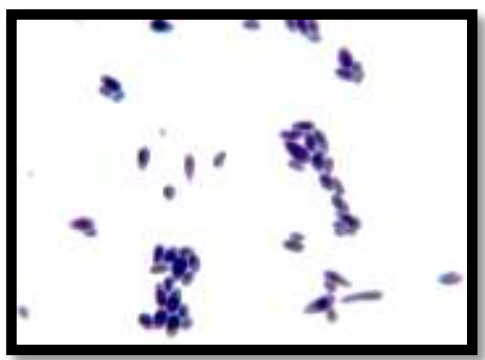

Microscopic picture of Candida tropicalis Using Gram stain

Figure (3): Gram staining of Candida species

Diagnosis by germ tube formation 


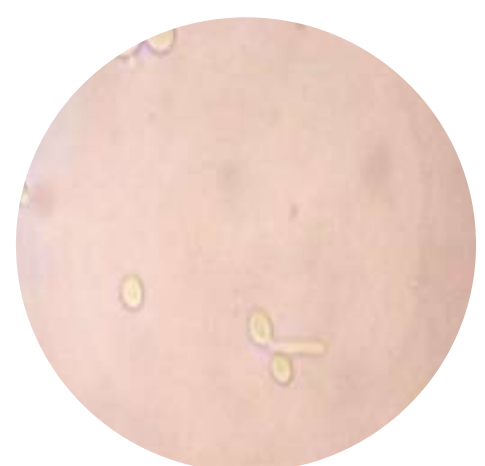

Figure (4): Candida albicans susceptibility to germ tube formation under microscope [40X]

\section{Diagnosis by CHROMO agar}

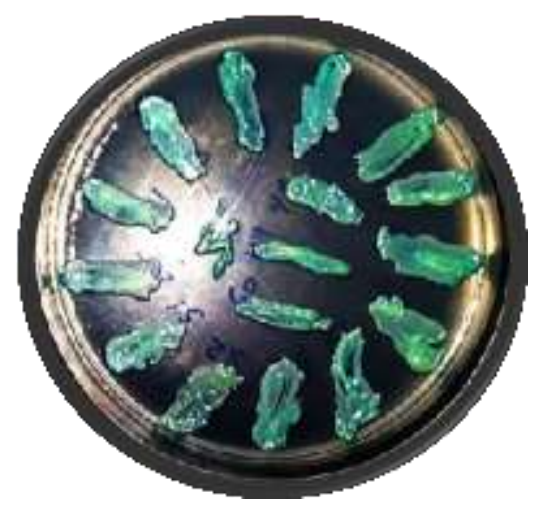

Figure (5): the growth of C. albicans on CHROMagar

Diagnosis of candida spp isolates using biochemical tests Diagnosis using the Vitek® apparatus

Yeast isolates were diagnosed as genus and species using biochemical tests. Positive results of the Vitek® system test showed that 115 species were diagnosed at the level of species and by (79\%). The other isolates could not be read by the Vitek®. This may be due to these isolates are contaminated with other bacterial or fungal species or may be a mixture of more than one type of candida (Mixed infection), making it difficult to diagnose and not read by Vitek apparatus.

However, through the morphology examination, it was found that the rest of isolates (30) isolates (21\%) belong to the genus Candida albicans, the most frequent yeast in Vitek examination is C.albicans. The number of isolates of this type (33) was isolated (29\%) and the total number of isolates of white candidiasis was $63(43 \%)$. This result is consistent with (3), they noted that the high frequency of C.albicans yeast in clinical samples compared with other species, Followed by C. guiliermondii (14) by (10\%), C. tropicalis (13) (9\%), C. krusei (12) (8\%), C. sphaeirica (10) (7\%), C. parapsilosis \& C. glabrata (9) (6\%), kefyer (7) (5\%), C. famata (5) (3\%) and C. norvegenesis (5) (3\%). The results showed in Table (2).

Table (2): Distribution of Candida infections in clinical samples

\begin{tabular}{|l|c|}
\hline \multicolumn{1}{|c|}{ Candida spp } & No. \\
\hline Candida albicans & 63 \\
\hline Candida glabrata & 9 \\
\hline Candida tropicalis & 13 \\
\hline Candida krusei & 12 \\
\hline Candida sphaeirica & 10 \\
\hline Candida parapsilosis & 9 \\
\hline Candida kefyer & 7 \\
\hline Candida guiliermondii & 14 \\
\hline Candida norvegenesis & 3 \\
\hline Candida famata & 5 \\
\hline
\end{tabular}




\section{Study effect of antibiotics on the Candida}

A total of 145 clinical isolates of Candida species were tested for antibiotics susceptibility against three antifungal drugs namely, Fluconazole, Clotrimazole and Nystatin, The antibiotics susceptibility of Candida species was tested by disc diffusion (DD), as (Figure 5), Candida spp 26 (18\%) percentage isolates were fluconazole, clotrimazole and nystatin resistant, and 48 (33\%) were S-DD for Fluconazole, and $71(49 \%)$ were susceptible to Floconazole, While the results show that 92 (63\%) were S-DD to Clotrimazole and 27 (19\%) were susceptible to Clotrimazole.

The test of Nystatin shows that $104(72 \%)$ were S-DD for Nystatin, While $15(10 \%)$ were susceptible to Nystatin (Table 3), these results agree with [25, 26, 27, 28, 29].

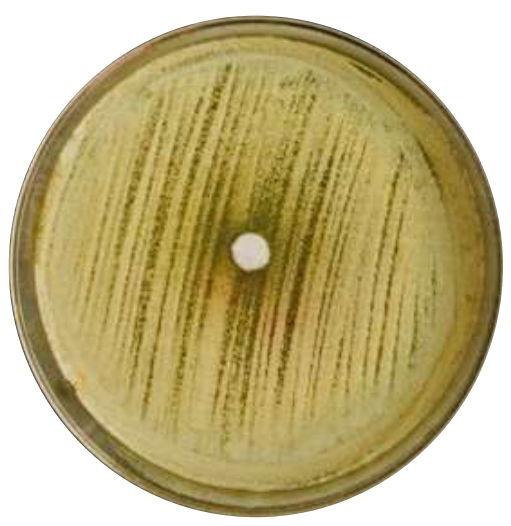

A

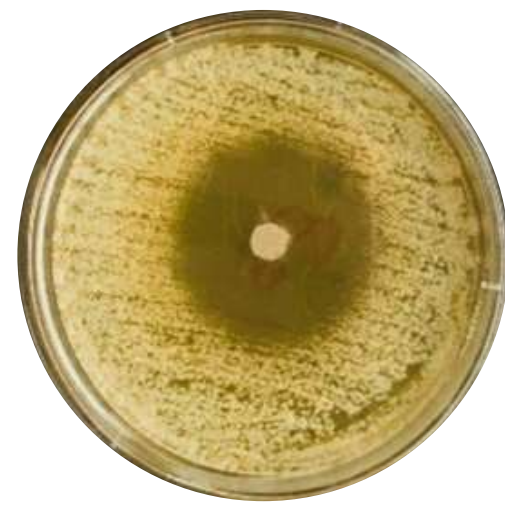

B

Figure (5): Antifungal susceptibility test against Candida species by disk diffusion method. [A]: Resistance [B]: Sensitive

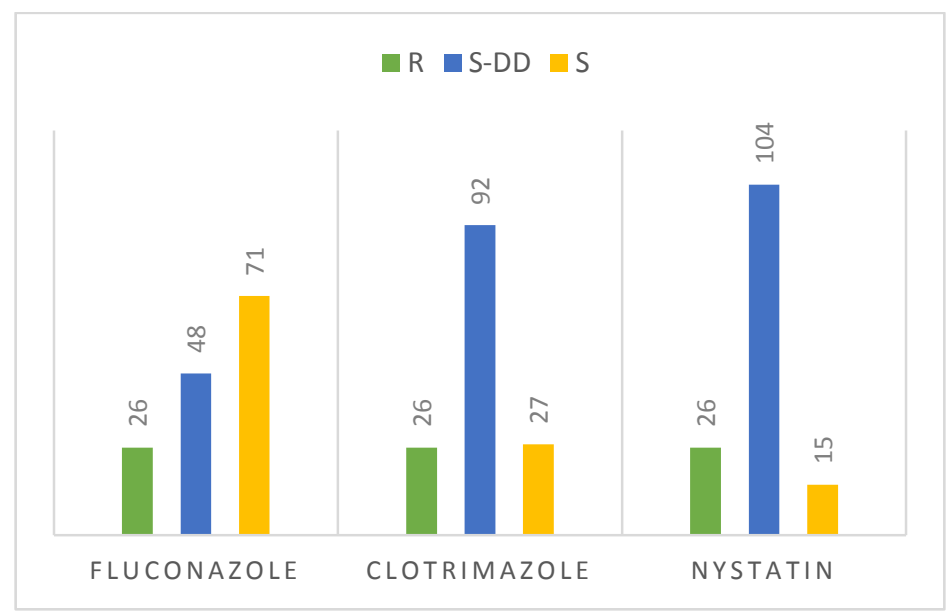

Figure (6): Antibiotics susceptibility test for Candida spp

\section{Percentage of [Sensitive and resistance] Candida species against different antifungal agents}

The results (Table 3) and (Figure 2) showed that (15) (54\%) isolates of Candida albicans were resistant, [33] (41\%) were isolated within normal growth and (13) (41\%) isolates were sensitive to the three antifungal which used in this study, while was (2) (7\%) resistance isolates from Candida glabrata, (7) (9\%) were in the normal inhibition from total (9) and did not show a sensitive isolation of antibiotics.

Candida tropicalis showed (2) (7\%) was resistant, (8) (10\%) percentage was grown in S-DD inhibition, and no sensitive.

Candida krusei was (3) (11\%) percentage are resistant, (5) (6\%) percentage was grown in S-DD inhibition, and sensitive is (4) (13\%) isolates.

Also the results showed Candida parapsilosis, kefyer and guiliermondii was (2) (7\%) are resistant, while the isolates Candida parapsilosis, kefyer was by (4) (5\%) and Candida guiliermondii was (9) (11\%) are grown in S-DD inhibition, the isolates which was sensitive are Candida kefyer and guiliermondii in (3) by (9\%).

For the isolates Candida norvegenesis was resistant in (1) by (4\%), (2) (2\%) are grown in S-DD, no sensitive. Candida famata showed (3) by (4\%) grown in S-DD and (2) (6) was sensitive. 


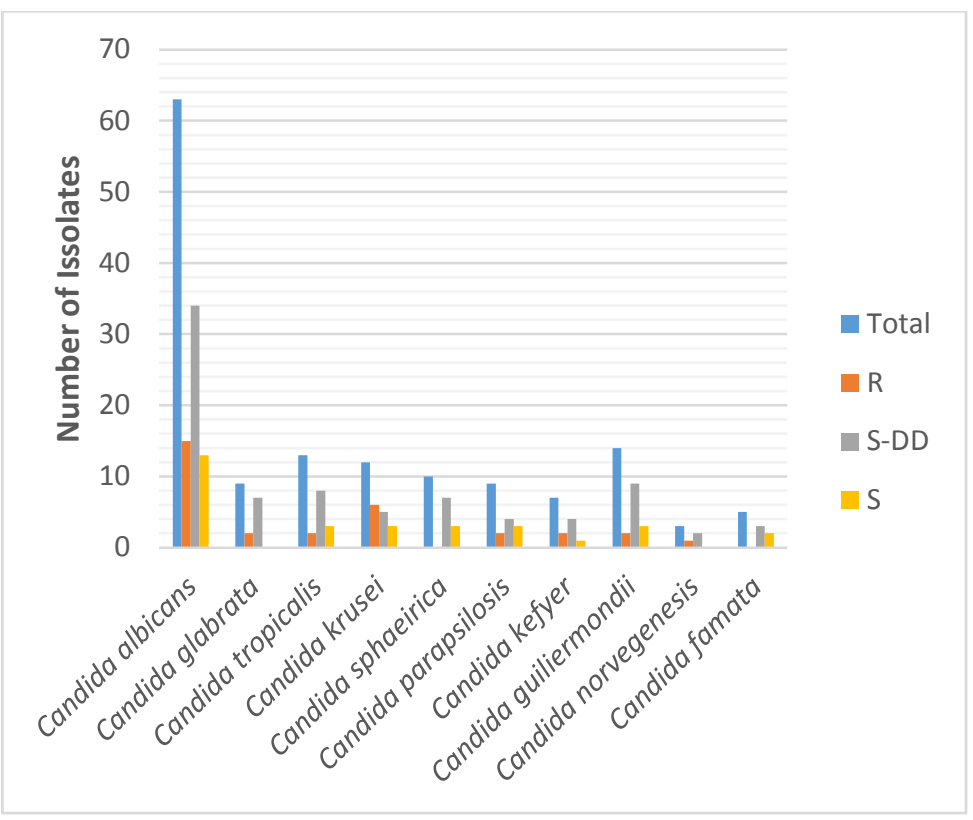

Figure (7): Types of Candida spp and antifungals susceptibility test for Candida spp

\section{Comparison of isolates by source of collection}

The samples were collected from different infectious areas of the body, taken from skin, oral thrux, lower respiratory tract, vaginal canal and urine. The inhibitory activity was tested on isolates under study. The results showed that most species isolated from the lower respiratory tract and vaginal tract were resistant to antibiotics, while the isolates from Skin, oral thrux and urine showed sensitivity to the antibiotics under study.

\section{Comparison of isolates isolated from vagina}

The results of the statistical analysis, as shown in (Table 3) showed that isolates isolated from the vaginal were effective resistance to the antifungals used comparison with criteria at (Table 3).

Table (3): Criteria of susceptibility for antifungals

\begin{tabular}{|l|c|c|c|c|}
\hline \multirow{2}{*}{ Anti-F } & \multirow{2}{*}{ Disk con. } & \multicolumn{3}{|c|}{ [Zone diameter ]mm } \\
\cline { 3 - 5 } FLU & $25 \mu \mathrm{g}$ & $\leq 14$ & $15-18$ & $\geq 19$ \\
\hline CLO & $10 \mu \mathrm{g}$ & $\leq 11$ & $12-19$ & $\geq 20$ \\
\hline NYS & $100 \mathrm{UN}$ & $\leq 16$ & $17-24$ & $\geq 25$ \\
\hline
\end{tabular}

The presence of effective resistance for candida spp to fluconazole by 26 with $18 \%$ percentage against all Candida species (Table 3).

Candida albicans $(8.8 \leq 14)$ this is the rate of inhibition diameter of Candida albicans. While the rate of inhibition diameters of the same type against Clotrimazole $(9.7 \leq 11)$ and $(12.7 \leq 16)$ for Nystatin. Whereas the statistical analysis showed that candida glabrata were resistance by $(13 \leq 14)$, candida guillermondii $(11 \leq 14)$, and candida parapsilosis were $(10 \leq 14)$.

The following species candida kefyer, candida krusei, candida famata and candida norvegensis showed no resistance against fluconazole.

The total Nystatin group gave inhibitory effect to most of the isolates used in the study, while most showed significant physical resistance, such as $C$. trpicalis, C. sphaerica, C. parapsilosis, C. guilliermonii, $C$. famata C.glabrata, C.parapsilosis, C.krusei, C.kefyr, and some isolates of C.albicans, according to the source of the combination, also showed sensitivity when treated with the group of polyneses, but some isolates of candidiasis showed high resistance against the group of polynes, especially that candidiasis characterized by defensive mechanisms and high virulence factors in against of antifungals, and the cause of resistance to isolates taken from the Lower respiratory and vagina use to frequent use Anti-fungal or vital irregularly, leading to adapt to the pathogenic on these high doses of antifungal and consequently become able to overcome these antibiotics effect in activating the ferocity of fungus by acquiring resistance to this type of antibiotics through generations. 
As for the isolates that showed their sensitivity to the antibody mentioned, the reason is that these fungi are present naturally in the areas mentioned, such as skin and oral mucus and genital canal vaginal, but it can turn into pathogen when the opportunity to seize the weakness of the immune system and others.

\section{Comparison of isolates isolated from respiratory tract}

The results showed that most of types as Candida albicans, Candida tropicalis, krusei, Candida glabrata and Candida guillermondii were resistance to all used antifungals in this study. While the other types as Candida sphaerica, Candida kefyer and Candida parapsilosis were sensitive and these results were similar for [30].

It was found that most of the isolates that showed resistance to the azole group (Fluoconazole and Clotrimazole) as well as the group of Polynes (Nystatin) were isolated from the lower respiratory tract. This supports the resistance of these species and their virulence. This is due to the widespread use of azoles at random without consulting a specialist, which increases the resistance of certain yeast species [31].

The randomly use of antibiotics used in the treatment of other infections in the body provides an opportunity for this disease and changes the microbiological environment, encouraging the colonization of Candida [32].

It may be caused by other chronic diseases that are responsible for reducing the immune system, creating an environment conducive to the stimulation and growth of these opportunistic organisms, and as a result they acquire actual resistance and increase their virulence [33,34].

These changes can be explained by pharmacological sensitivity with the time, the ability of yeast to tolerate the toxic effects of antimicrobial resistance and its ability to develop some resistance mechanisms. The differences in the results of the current study with the results of other studies in developed countries based on the environmental location and excessive intake of antimicrobial agents may explain the transmission of genetic resistance between resistant isolates to sensitive isolates Due to unhealthy and personal hygiene practices, including the introduction of sewage into drinking water, leading to the transmission of resistance genes [33,35].

The resistance of isolates from the lower respiratory tract is due to the frequent use of antifungal or non-systemic antibiotics, which has resulted in the adaptation of the pathogens to these high doses of antibiotics. As a result, they have been able to overcome the effect of these antibiotics in stimulating the virulence of fungi by acquiring resistance to this type of antigens throughout generations.

\section{References}

1. Al-Tekreeti AR, Al-Halbosiy MMF, Dheeb BI, Hashim AJ, Al-Zuhairi AFH. Molecular identification of clinical Candida isolates by simple and randomly amplified polymorphic DNA-PCR. Arab J. Sci. Eng. (2017); DOI 10.1007/s13369-017-2762-1.

2. Ayesha A, Prevalence, Antifungal Susceptibility and Biofilm Characterization of Candida Species Isolated from Tertiary Care Hospitals, 2016; Quaid-i-Azam University, Islamabad, Pakistan.(2018); 1-22.

3. Rossoni RD, Barbosa JO, Vilela SFG, dos Santos JD, de Barros PP, Prata MC, de A, Junqueira JC. Competitive Interactions between C. albicans, C. glabrata and C. krusei during Biofilm Formation and Development of Experimental Candidiasis. (2015); PLOS ONE, 10(7).

4. Dheeb BI, Al-Halbosiy MMF, Al lihabi RK, Khashman BM. The effects of Rubus idaeus extract on normal human lymphocytes and cancer cell line. BMC Genomics17. (Suppl 6). (2016); p. 19.

5. Nouri MA, Al-Halbosiy MMF, Dheeb BI, Hashim AJ. Cytotoxicity and genotoxicity of gliotoxin on human lymphocytes in vitro. Journal of K S U - Science. (2015); 27, 193-197.

6. Cornet M, Sendid B, Fradin C, Gaillardin C, Poulain D, Nguyen H. -V. Molecular Identification of Closely Related Candida Species Using Two Ribosomal Intergenic Spacer Fingerprinting Methods. The Journal of Molecular Diagnostics. (2011); 13(1), 12-22.

7. Hussain AF, Sulaiman GM, Dheeb BI, Hashim AJ, Seddiq SH. Improving conditions for gliotoxin production by local isolates of Aspergillus fumigatus. Journal of biotechnology research center. (2017); 11(2):14-24.

8. Rassin NK, Nemat JA, Dheeb BI. Molecular Identification of Aspergillus fumigatus Using ISSR and RAPD Markers. Iraqi Journal of Science. (2015); 56 (4A), 2788-2797.

9. Nobile CJ, Johnson AD. Candida albicans biofilms and human disease. Annu Rev Microbiol . (2015); 69:71-92.

10. Claudia S, Darío L. Candida Infections, Causes, Targets, and Resistance Mechanisms: Traditional and Alternative Antifungal Agents. (2013); 23(2), 253-273.

11. Achkar JM, Fries BC. Candida Infections of the Genitourinary Tract. Clinical Microbiology Reviews. (2010); 23(2), 253-273.

12. Dheeb BI, Al-Mashhadani II, Ismail EN, Majeed SM, Majeed DM. A study of the Expression of Aflatoxin B1 Regulatory Gene in Clinical and Environmental Aspergillusflavus using Real-time PCR. I J S: B A R. (2014). 17 (1), 417-427. 
13. Ibrahim IM, Iftikhar M, Ali IM, Dheeb BI, Abbas QA, Ramizy A, Eisa MH, Aljameel AI. Antifungal activity of wide band gap Thioglycolic acid capped ZnS:Mn semiconductor nanoparticles against some pathogenic fungi . Materials Science and Engineering. (2017); 73:665-669.

14. Bander KI, Mohammed SH, Thalij KM, Dheeb BI. Survey Study of theAllergic Fungi in Kirkuk Area and Use Molecular Detection for Identification. I J S: B A R. (2015); 19(1):383-397.

15. Ruhnke M. Clinical Syndromes: Candida and Candidosis. Clinically Relevant Mycoses. (2018); 45-75.

16. Dheeb BI, Al-Mudallal NH, Salman ZA, Ali M. The Inhibitory Effects of Human, Camel and Cow's Milk against Some Pathogenic Fungi in Iraq. Jordan Journal of Biological Sciences. (2015); 8(2) 89 - 93.

17. Lohse MB, Gulati M, Johnson AD, Nobile CJ. Development and regulation of single- and multi-species Candida albicans biofilms. Nature Reviews Microbiology. (2017); 16(1), 19-31.

18. Gow NAR, Netea MG. Medical mycology and fungal immunology: new research perspectives addressing a major world health challenge. Philosophical Transactions of the Royal Society B: Biological Sciences. (2016); 371(1709).

19. Brown GD, Denning DW, Gow NAR, Levitz SM, Netea MG, White TC. Hidden killers: human fungal infections. Sci. Transl. Med. (2012); 19, 165 rv13.

20. Casadevall A, Pirofski LA. The damage-response framework of microbial pathogenesis. Nat. Rev. Microbiol. (2003); 1, 17-24.

21. El-Hilali F, El-Hilali H, Dheeb BI, Traore BM, Messouak M, Mazouz H, Moumni M, Belgacem FBM, El-Mowafy AM. Blood Transfusion Utility DuringCardiopulmonary Bypass and Correlation with Key-Biochemical Laboratory Findings: A New Approach to Identify Preventive and Risk Factors (1Year Practice at University Hospital Hassan-II of Fez). Biochem Anal Biochem. (2016); 5:3 DOI: 10.4172/2161-1009.1000290.

22. Sardi JCO, Pitangui NDS, Gullo FP, Fusco-Almeida AM, Giannini MJSM. A mini review of Candida species in hospital infection: Epidemiology, virulence factor and drugs resistance and prophylaxis. Trop Med Surg. (2013); 1: 141.

23. Hilmar W, Michaelm B, Jenny E, Lea C, Danuta S, Yvette M, Richard P, Harald S. Nosocomial bloodstream infections due to Candida spp. In the USA: species distribution, clinical features and antifungal susceptibilities. International Journal of Antimicrobial Agents, (2014); 43: 78-81.

24. Abdulbaqi NJ, Dheeb BI, Irshad R. Expression of Biotransformation and Antioxidant Genes in the Liver of Albino Mice after Exposure to Aflatoxin B1 and an Antioxidant Sourced from Turmeric (Curcuma longa). Jordan Journal of Biological Sciences. (2018); 11(2) 89 - 93.

25. Koneman EW, Roberts GD. Practical laboratory Mycology. 3rd edition, Williams and Williams, USA.(1985); 211 .

26. Larone DH. Medically important fungi: aguide and identification. (3th Ed.) Asm. Press, washtngton, D.C. (Abst.). (1995).

27. Kumar MB, Edward CK. Robbins and Cotran Review of Pathology, Elisever. (2014); 6-7.

28. Kwon-Chung KJ, Bennett JE. Medical Mycology.Lea and Febiger, Philadelphia. (1992).

29. Mahmoudabadi Ali Z, Zarrin M, Beheshti FM. Antifungal Susceptibility of Candida Species Isolated From Candiduria, Jundishapur J Microbiol. (2013); 6(1): 24-28.

30. McGinnis MR. Laboratory hand book of medical mycology. Acadmic press. New York. (1980); P: 356.

31. Hospenthal DR, Beckius ML, Floyd KL, Horvath LL, Murray CK. Presumptive identification of Candida species other than C. albicans, C. krusei, and C. tropicalis with the chromogenic medium CHROMagar Candida. Ann Clin Microbiol Antimicrob. (2006); 3(5): 1-10.

32. Osaigbovo I, Lofor P, Oladele R. Fluconazole Resistance among Oral Candida Isolates from People Living with HIV/AIDS in a Nigerian Tertiary Hospital. J. Fungi. (2017); 3:8.

33. AL-Ani , Zouhair I, AL-Maliki Rehab S. Antifungal resistance of Candida species isolated from Iraqi women infected with vulvovaginal Candidiasis .QMJ VOL. (2011); 7 (11):117-127.

34. Hussain AF, Sulaiman GM, Dheeb BI, Hashim AJ. Histopathological changes and expression of transforming growth factor beta (TGF- $\beta 3$ ) in mice exposed to gliotoxin. Journal of K S U - Science. (2018); 27, 193-197.12.

35. Dheeb BI, Al-dujayli SMA, Ibrahim IM, Abbas QA. Study the Antifungal Activity of ZnS:Mn Nanoparticles Against Some Isolated Pathogenic Fungi. Journal of Physics: Conference Series. (2019); 1178, 46-52. 